TRADE NEWS FOCUS
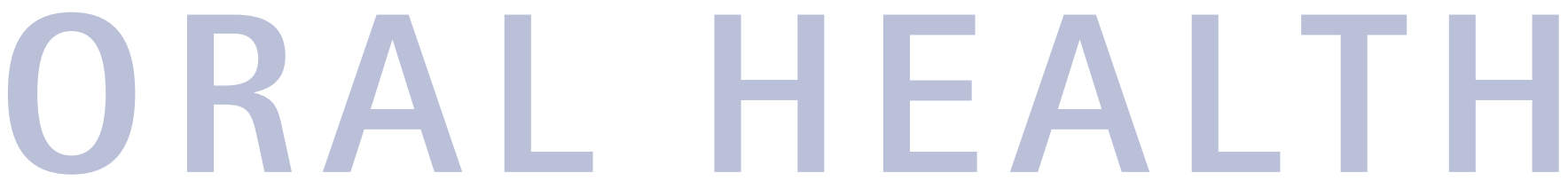

\section{Cold sore advice}

Understanding the Herpes Simplex Virus (HSV) can help dentists to avoid cross infection in their practice, and also to support and advise patients who suffer from cold sores.

The Cold Sore Information Bureau (CSIB) has developed a new best practice guide to help dentists manage issues surrounding the treatment and support of patients who experience cold sores. The guide is based on the knowledge and experience of the CSIB's independent dental panel and provides practical advice for both dentists and patients.

The CSIB has also produced a bespoke dental pack for use in surgery, which includes a copy of the best practice guide; a colourful and informative A3 poster providing advice for patients for display in surgery waiting rooms, examples of CSIB patient leaflets, developed to provide cold sore sufferers with help and advice on the condition, plus an application form to request further copies of these leaflets for the surgery.

To request a free dental pack, send an email to the CSIB at CSIB@harvardhealth.co.uk. Please entitle the email CSIB Best Practice Guide and remember to include your name, address and practice details in the email. Or write to CSIB Dental Pack, c/o Harvard Health, Suite 2, Cobb House, Oyster Lane, Byfleet, Surrey KT14 7DU.

Reader response number 54

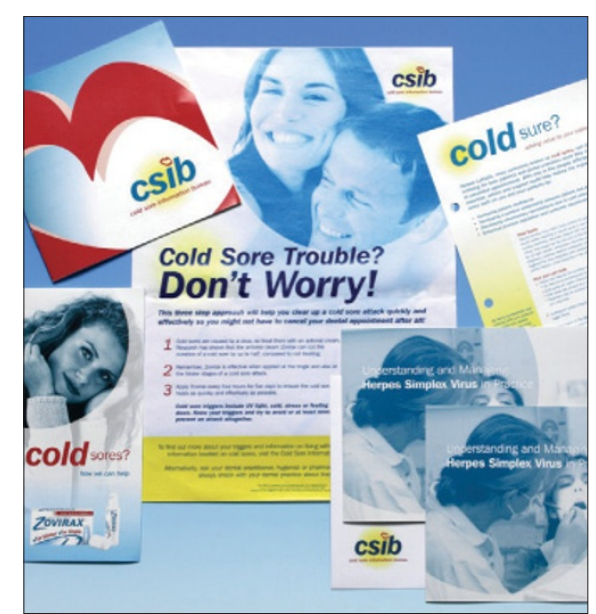

\section{Healing patients quicker}

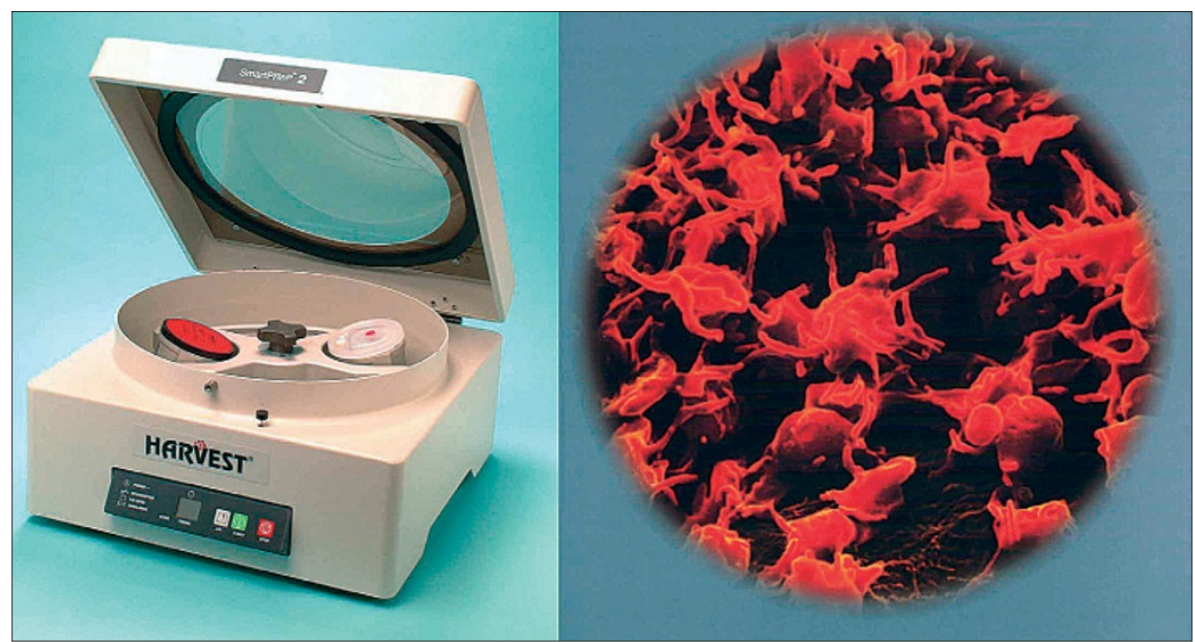

The SmartPReP ${ }^{\circledR} 2$ APC+ System is the optimum way to harvest platelet rich plasma for APC+ Treatment, according to Haley Ltd. SmartPReP ${ }^{\circledR} 2$ is the table top version of the innovative SmartPReP APC+ System.

Its compact design saves surgery space and enables the successful harvesting of APC+ using only a small sample of the patient's blood. Enabling clinicians to provide patients' with all the benefits of APC+ treatment quickly and easily, it is patient friendly and cost effective for the clinician.

It can be utilised in a variety of treatments including implantology, oral surgery and periodontology. The benefits include enhanced bone growth and matu-

ration, graft stabilisation, wound sealing, wound healing and haemostasis.

The combination of these benefits helps to reduce post-operative swelling, pain and risk of infection.

Independent research has shown that treatment with Autologous Platelet Concentrate $(\mathrm{APC}+)$ will enhance a patient's oral health in a variety of clinical situations including following basic minor oral surgery, treatment of periodontal defects, implantology, immediate denture provision and more. Results have also shown that following its application, healing is accelerated, pain is reduced and autogenous bone is retained.

Reader response number 55

\title{
Confidence with Poli-Grip
}

The Poli-Grip Range consists of Poli-Grip Ultra, Poli-Grip Fresh Gel, and Poli-Grip Flavour Free which have a unique formula that offers lasting all day hold. Bite force is increased by almost 40 per cent and food particles that become trapped under the plate are reduced by up to two thirds.

Denture Care Packs from Poli-Grip are available free to both dentists and patients, and include a denture holder and bath, QEtA patient information leaflet, Poli-Grip and Dentu-Creme product samples and a

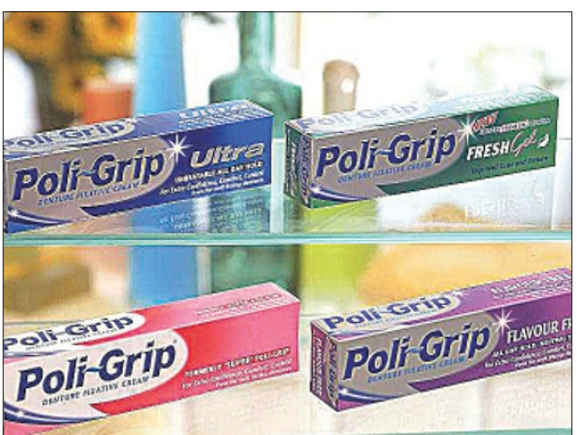
money off voucher. This can be ordered by calling 02080474048 . Reader response number 56 


\section{A touch of Hollywood}

The Beverly Hills Formula range aims to provide innovative, high quality, niche products in the oral hygiene market. Every bottle of the Beverly Hills Formula Mouthrinse has a unique anti-bacterialtongue scraper.

The toothpaste is proven to effectively remove tooth staining, while maintaining extremely low abrasion. Both the toothpaste and mouthrinse help restore teeth to their natural colour while protecting against decay, plaque and gum disease. Tests conducted by the BBC Watchdog programme revealed that Beverly Hills Formula Toothpaste removed over 90 per cent of staining.

Reader response number 57

\section{Colour coded pastes}

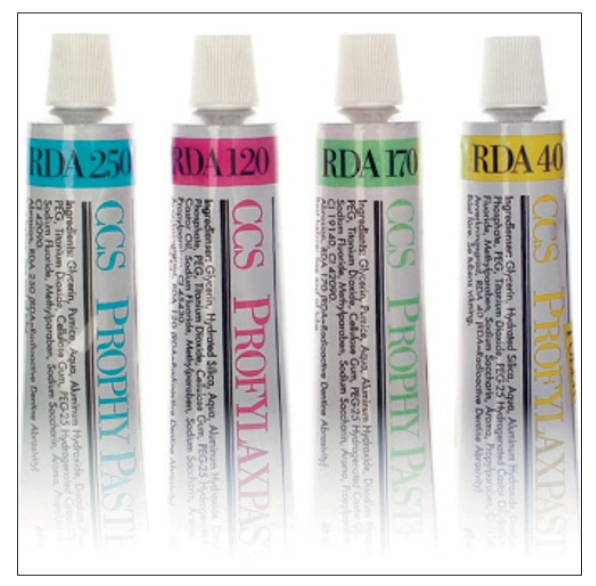

The CCS Colour Coded Fluoride Prophylaxis Paste from SDI is formulated to be non-splattering, does not dry out, liquefy or separate and has a long shelf life. Easy to rinse off, it replaces fluoride as it is polished off and does not leave an oily residue.

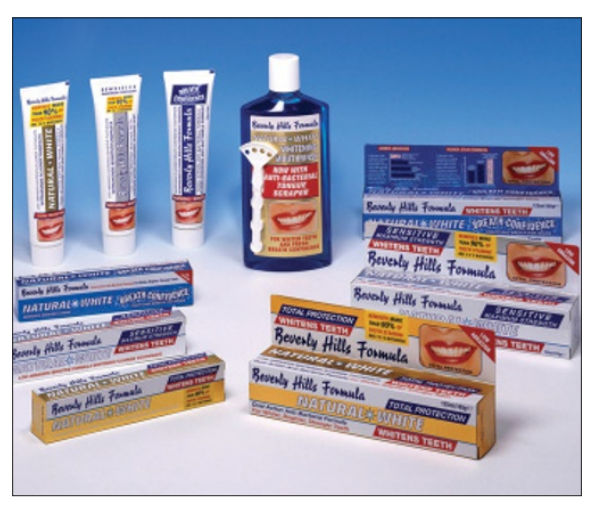

CCS Prophy Paste is available in four colours for easy identification of the appropriate grit size and indication.

The coarse blue paste has a $125 \mu \mathrm{m}$ grit size and is designed for use on heavily discoloured surfaces and thick layers of plaque.

The medium green paste has a 70 $\mu \mathrm{m}$ grit size and is designed for thickly layered plaque and moderately discoloured teeth.

The fine red paste has a $20 \mu \mathrm{m}$ grit size and is a universal paste designed for smaller amounts of plaque, slightly discoloured teeth and root surfaces.

The extra fine yellow paste has a $2 \mu \mathrm{m}$ grit size and is designed for polishing teeth and restorations. It gives an extremely smooth finish.

Economically priced, the complete array of CCS Colour Coded Fluoride Prophylaxis Pastes are detailed with the Trycare Catalogue.

Reader response number 58

\section{Attachment system for overdentures}

The Zest Locator self-aligning attachment system for implant and root retained overdentures can help ease difficulties from ill-fitting dentures, according to Haley Ltd.

It is designed to reduce the height of abutment restorations on all brands of endosseous implants. With a total attachment height (male plus female) of only $3.17 \mathrm{~mm}$ for an externally hexed implant, the Locator saves a minimum of $1.68 \mathrm{~mm}$ to $3.05 \mathrm{~mm}$ of inter-occlusal space compared with other implant overdenture attachments.

To facilitate greater flexibility, each set of Locator attachments contains a choice of three colour coded removable nylon caps. The original white cap has a strong 5.0 pound holding force, the pink cap a medium 3.0 pound retention and the blue cap a light 1.5 pound retention. They enable the dentist to select the right choice of strong, medium or light retention according to the needs of the patient.

Reader response number 59

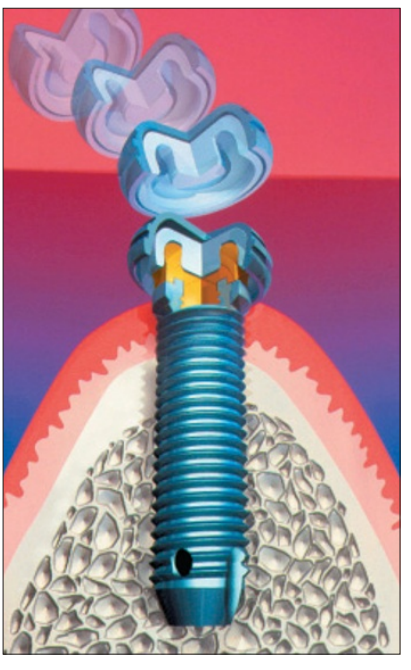

